\title{
Determination of humidity and temperature fluctuations based on MOZAIC data and parametrisation of persistent contrail coverage for general circulation models
}

\author{
K. M. Gierens ${ }^{1}$, U. Schumann ${ }^{1}$, H. G. J. Smit ${ }^{2}$, M. Helten ${ }^{2}$, G. Zängl ${ }^{1}$ \\ ${ }^{1}$ DLR, Institut für Physik der Atmosphäre, Oberpfaffenhofen, D-82230 Weßling, Germany \\ ${ }^{2}$ Forschungszentrum Jülich, Institut für Chemie und Dynamik (ICG2), Postfach 1913, D-52425 Jülich, Germany
}

Received: 21 October 1996 / Revised: 7 February 1997 / Accepted: 24 February 1997

\begin{abstract}
Humidity and temperature fluctuations at pressure levels between 166 and $290 \mathrm{hPa}$ on the grid scale of general circulation models for a region covered by the routes of airliners, mainly over the Atlantic, have been determined by evaluation of the data obtained with almost 2000 flights within the MOZAIC programme. It is found that the distributions of the fluctuations cannot be modelled by Gaussian distributions, because large fluctuations appear with a relatively high frequency. Lorentz distributions were used for the analytical representation of the fluctuation distributions. From these a joint probability distribution has been derived for simultaneous temperature and humidity fluctuations. This function together with the criteria for the formation and persistence of contrails are used to derive the maximum possible fractional coverage of persistent contrails in a grid cell of a GCM. This can be employed in a statistical formulation of contrail appearance in a climate model.
\end{abstract}

\section{Introduction}

Contrails, like natural clouds, modify the radiation budget of the Earth-atmosphere system. The question whether contrails may have an influence on the climate has so far been addressed by means of principle studies (Graß1, 1990) or 1-D radiation-convection models (e.g. Fortuin et al., 1995). In such studies only the direct effect of local radiative and convective processes can be investigated, whereas other feedback mechanisms must be neglected. Ponater et al. (1996) first estimated the contrail effect on climate using a 3-D global circulation model (GCM), namely the German climate community model ECHAM (Roeckner et al., 1992). It has been

Correspondence to: K. M. Gierens demonstrated that contrails have the potential for a climate change. However, Ponater et al.'s (1996) study did not provide a quantitative assessment because contrails were inserted into the climate model without regard to the meteorological conditions. Therefore, it is desirable to put the contrail parametrisation in GCMs onto a sounder footing by taking into account the thermodynamic criteria for the formation and persistence of contrails (Schmidt, 1941; Appleman, 1953; Schumann, 1996).

The Schmidt-Appleman criterion states that an aircraft produces a contrail only when a supersaturated state (with respect to the liquid phase) is reached during the mixing of the exhaust gas with the ambient air. This criterion is a local one, i.e. it relates the possibility of contrail formation to the local thermodynamic state. On a scale that is resolved by a GCM, however, such a local criterion would be of questionable applicability because the GCM provides only grid cell mean values of the thermodynamic state. A statistically more satisfying treatment of contrail formation can be obtained when the fluctuations of the state variables around their gridscale mean values are known. Then the rigid yes/noalternative of the Schmidt-Appleman criterion can be replaced by a probability for contrail formation, which can be translated into a fractional coverage with respect to one grid cell of a GCM. It is one aim of this study to determine the fluctuations of temperature and relative humidity on a scale that is resolved by a GCM. This will be achieved by an evaluation of the data obtained during the MOZAIC programme (Marenco et al., 1994).

The objective of the MOZAIC programme (Measurement of OZone on Airbus In-service airCraft) is to perform large scale and continuous measurements of ozone and water vapour aboard in-service aircraft in order to realise a sound data base of atmospheric composition and trace species chemistry at current aircraft cruise altitudes. For this purpose five automatic measuring units have been installed on commercial airliners. These units not only measure ozone and water vapour concentration, but also record the meteorologi- 
cal quantities temperature and pressure. MOZAIC therefore provides a dataset of meteorological variables that is unique with respect to spatial and temporal coverage of the major flight routes.

In this study a parametrisation of persistent contrail coverage for GCMs is developed. We will carry out the parametrisation for a specific GCM, namely ECHAM in T42 resolution. The setup of the parametrisation for other GCMs or other resolutions is then straightforward. In order to achieve this objective, we first use the MOZAIC data to determine the spatial distribution of temperature and humidity fluctuations on a $300 \times 300$ $\mathrm{km}^{2}$ scale which corresponds to the size of one grid cell in T42 resolution (Sect. 2). The fluctuation distributions together with the local criteria for the formation and persistence of contrails then allow us to compute the maximum fractional coverage of persistent contrails in an ECHAM grid cell (Sect. 3). In this fraction of the considered cell the air is cold and moist enough to allow contrails to persist. These areas can be interpreted as the moist lenses where contrails appear in groups (Bakan et al., 1994). The methods and results are discussed in Sect. 4 and summarized in Sect. 5.

\section{Evaluation of MOZAIC data}

\subsection{General matters}

MOZAIC covers the time from July 1994 until December 1995, however we constrain our analysis to the year 1995 in order to have a complete annual cycle. The data bank contains data from almost 2000 flights in 1995. From the data base we obtain 1 min averages of pressure $p$, temperature $T$, and relative humidity $R H$ at the current flight position (longitude $\lambda$, latitude $\beta$, altitude $z$ ). Each flight position $(\lambda, \beta)$ belongs to a certain grid cell in the ECHAM general circulation model (Roeckner et al., 1992). In T42 resolution the zonal direction is divided into 128 sectors, the meridional direction into 64 segments, giving $128 \times 64=8192$ grid cells. The data points are sorted into the ECHAM altitude layers by means of the measured static pressures $p$, since ECHAM uses a hybrid $\sigma-p$ altitude coordiante which at cruise flight levels is nearly equivalent to a pure pressure coordinate. We constrain the evaluation on the altitude range 8-14 $\mathrm{km}$, since this range covers the usual cruise flight levels of civil aviation. The corresponding pressures in ECHAM range from 122 to $367.5 \mathrm{hPa}$, which are represented by levels 6 to 9 (the counting starts at the top of the atmosphere). In level 6 , there were no data at all. In the lowest considered level 9, the aircraft usually were in ascent after take off or in descent before landing. Our analysis is therefore effectively constrained to levels 7 and 8, which corresponds to a pressure range 166-290 $\mathrm{hPa}$. The mid-level pressures are $190 \mathrm{hPa}$ for level 7 and $250 \mathrm{hPa}$ for level 8. The mean accuracy of the 1995 MOZAIC RH measurements is $\pm 6 \%$ at $290 \mathrm{hPa}$ and $\pm 35 \%$ at $166 \mathrm{hPa}$ relative to the measured value. The accuracy of the temperature measurements is $\pm 0.5 \mathrm{~K}$.

\subsection{Indicator variables}

From the physical variables $p, T$, and $R H$, we compute indicator variables, viz.

$C=1$ if the Schmidt-Appleman criterion is fulfilled, otherwise $C=0$

$I C=1$ if ice saturation is reached, otherwise $I C=0$

$W C=1$ if water saturation is reached, otherwise $W C=0$

$P C=1$ if contrails can persist, otherwise $P C=0$

There are the obvious logical relations:

$I C=0 \Rightarrow W C=0, W C=1 \Rightarrow I C=1$,

and

$P C=(1-W C) \cdot I C \cdot C$,

since contrail persistence requires ice saturation and the Schmidt-Appleman criterion to be fulfilled. Situations with $R H$ exceeding water saturation usually imply flight in clouds and are not considered contrail cases.

For the evaluation of the Schmidt-Appleman criterion we need some assumptions about properties of the kerosene burned in the engines and on the propulsion efficiency $\eta$ of an average (present day) aircraft (see Busen and Schumann, 1995; Schumann, 1996). We assume that the water vapour emission index $\mathrm{EI}_{\mathrm{H}_{2} \mathrm{O}}=1.25 \mathrm{~kg} / \mathrm{kg}$ fuel and that the combustion heat is $43 \mathrm{MJ} / \mathrm{kg}$ fuel. Furthermore, we assume $\eta=0.3$ which is a typical value for modern bypass engines (Schumann, 1996). The critical temperature of the Schmidt-Appleman criterion varies by $0.7 \mathrm{~K}$ for a variation of $\Delta \eta=0.05$ (Schumann, 1996).

The indicator variable $P C$ is a function of only temperature and relative humidity if the pressure is held fixed, as e.g. on a constant flight level. We may thus consider a $(T, R H)$-diagram and determine the part of the $(T, R H)$-space where $P C=1$. This area has a rather simple shape: it is bounded on three sides by nearly straight lines (Fig. 1). The two boundaries at low $R H$ (about $60 \%$ ) and at $R H=100 \%$ are due to the ice and water saturation conditions. The boundary at about $T \approx-45^{\circ} \mathrm{C}$ is due to the Schmidt-Appleman criterion (see the logical relation for $P C$ already mentioned). Only the latter boundary depends on the chosen pressure. In Fig. 1 we have plotted the Schmidt-Appleman boundary for the two mean pressures of the ECHAM levels 7 and 8 , i.e. 190 and $250 \mathrm{hPa}$. The variation of the SchmidtAppleman boundary with pressure within one ECHAM layer is of the order $1 \mathrm{~K}$.

Although it would be interesting to show statistics of, e.g. ice saturation or conditions leading to persistent contrails, we dispense with it for the present study, since it is not necessary for the purpose of the desired parametrisation.

\subsection{Fluctuations in ECHAM grid cells}

A single ECHAM-T42 grid cell is about $300 \times 300 \mathrm{~km}^{2}$ in size. The prognostic physical variables must therefore be regarded as mean values for such a cell and for the 


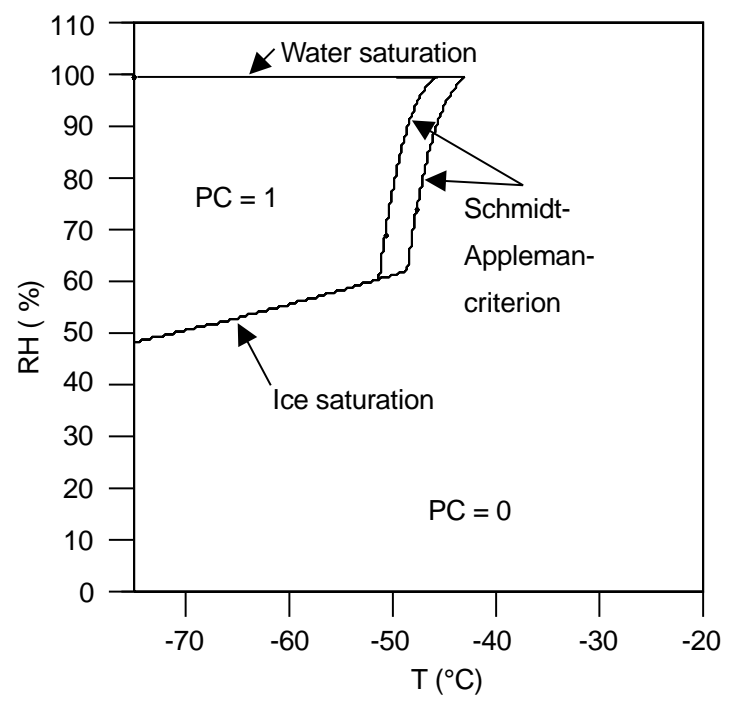

Fig. 1. For a constant pressure (as e.g. on a constant flight level) the possibility for persistent contrails development depends only on temperature and relative humidity. Persistent contrails are possible in the area indicated by $P C=1$. This area is bounded on three sides: by low $R H$ because of the ice saturation condition, at $R H=100 \%$ because of the water saturation condition, and at high $T$ because of the Schmidt-Appleman criterion. The latter criterion depends on the pressure. The two boundaries are drawn for 190 (left curve) and $250 \mathrm{hPa}$ (right curve)

time step (21 min). For the simulation of subgrid-scale phenomena like clouds (including contrails), the fluctuations of physical variables around their mean values should be known. Here we can determine such fluctuations from the data on the MOZAIC data bank. Since the pressure is mostly more or less constant during flights, it is not possible to determine pressure fluctuations. However, since the vertical ECHAM coordinate assumes constant pressure and in view of the small vertical pressure variation within one ECHAM altitude layer, the determination of horizontal pressure variations does not seem necessary. Only fluctuations of temperature and relative humidity are thus considered.

As an illustration of such fluctuations consider Fig. 2 and 3 where we have plotted on the world map the "mean standard deviation" of temperature and relative humidity, as obtained from the data bank. The term mean standard deviation means that we have first determined standard deviations for each pixel and each flight separately. This has been done only when eight or more data points (i.e. one minute average values of humidity and temperature) were obtained in the grid cell under consideration. Eight data points correspond to a distance of about $100 \mathrm{~km}$ which we considered minimum to be representative for grid cells with $300 \mathrm{~km}$ side length. Measurements in cloudy cells $(R H \geq 100 \%)$ have been ignored. After that, we have averaged the individual standard deviations when more than 10 flights crossed the pixel in 1995. Averaging this way avoids contamination of the results with annual or day by day variations. The mean standard deviation is, therefore, a measure of instantaneous fluctuations of temperature and relative humidity. The maps show that instantaneous temperature fluctuations on the $300 \times 300$ $\mathrm{km}^{2}$ scale are less than $1 \mathrm{~K}$ almost everywhere. They are slightly larger $(0.4-0.8 \mathrm{~K})$ over the Atlantic than in the tropics $(0-0.4 \mathrm{~K})$, presumably because the meridional gradient of the zonal mean temperature is much larger in mid-latitudes than in tropical regions (see, e.g. Fig. 10.11 in Holton, 1979). Accordingly, an aircraft flying at a constant pressure level and with a meridional component in its flight route passes through a larger temperature change over the Atlantic than in the tropics. Apparent maxima of temperature fluctuations at the endpoints of flight routes are caused by ascent and descent near airports. For the evaluation only data pertaining to constant pressure levels are used. Fluctuations of relative humidity are typically of the order 4 $6 \%$ over the North Atlantic, but in tropical regions they reach $10 \%$, presumably because of the strong convection there.

We can determine the distribution law for instantaneous temperature and relative humidity fluctuations. Again, we first consider each flight separately. We collect all data points that pertain to one ECHAM grid cell and compute mean temperature $\langle T\rangle$ and mean relative humidity $\langle R H\rangle$ for this cell. The number of data points for the calculation of these mean values is typically around 20 . Grid cells with fewer than six data points and data points inside clouds $(R H \geq 100 \%)$ are ignored. Furthermore, we record the individual deviations $\delta T_{v}=T_{v}-\langle T\rangle$ and $\delta R H_{v}=R H_{v}-\langle R H\rangle$ ( $v$ is the data point number). Strictly speaking, the computed mean values and fluctuations refer to a line through a cell, not to the cell as an area or a volume. This may cause an underestimation of the width of the fluctuation distribution within the grid cell. Ideally, we would like to have (nearly) simultaneous measurements at many locations throughout a grid cell. Since only five aircraft were involved in the MOZAIC programme, the data base does not contain such details. So there is no other choice than to relate the computed mean values to the prognostic ECHAM variables and to consider the recorded fluctuations as characteristic for the grid cell as a volume. The fluctuations are sorted in bins of $0.04 \mathrm{~K}$ and $0.2 \%$, respectively; the mean values are not used further.

The fluctuation data for all grid cells and all 1924 flights in 1995 are then collected. Thus, we do not retain any information about spatial and temporal variation of the fluctuation distributions. This is not necessary since GCM modellers prefer to have one parametrisation for the whole globe and the whole year instead of dealing with many parametrisations for the same purpose. The reason is, that generally a parametrisation involves tuning factors, and the number of tuning factors should be as small as possible. Therefore, we have only retained the altitude information in the data and analysed the fluctuations for ECHAM levels 7 and 8 separately. The distributions of temperature and relative humidity fluctuations are displayed in Fig. 4 (upper and middle panel) as dots. It can be seen that these distributions cannot be modelled by a Gaussian distribution because large fluctuations have a relatively high probability. The 
Level 7
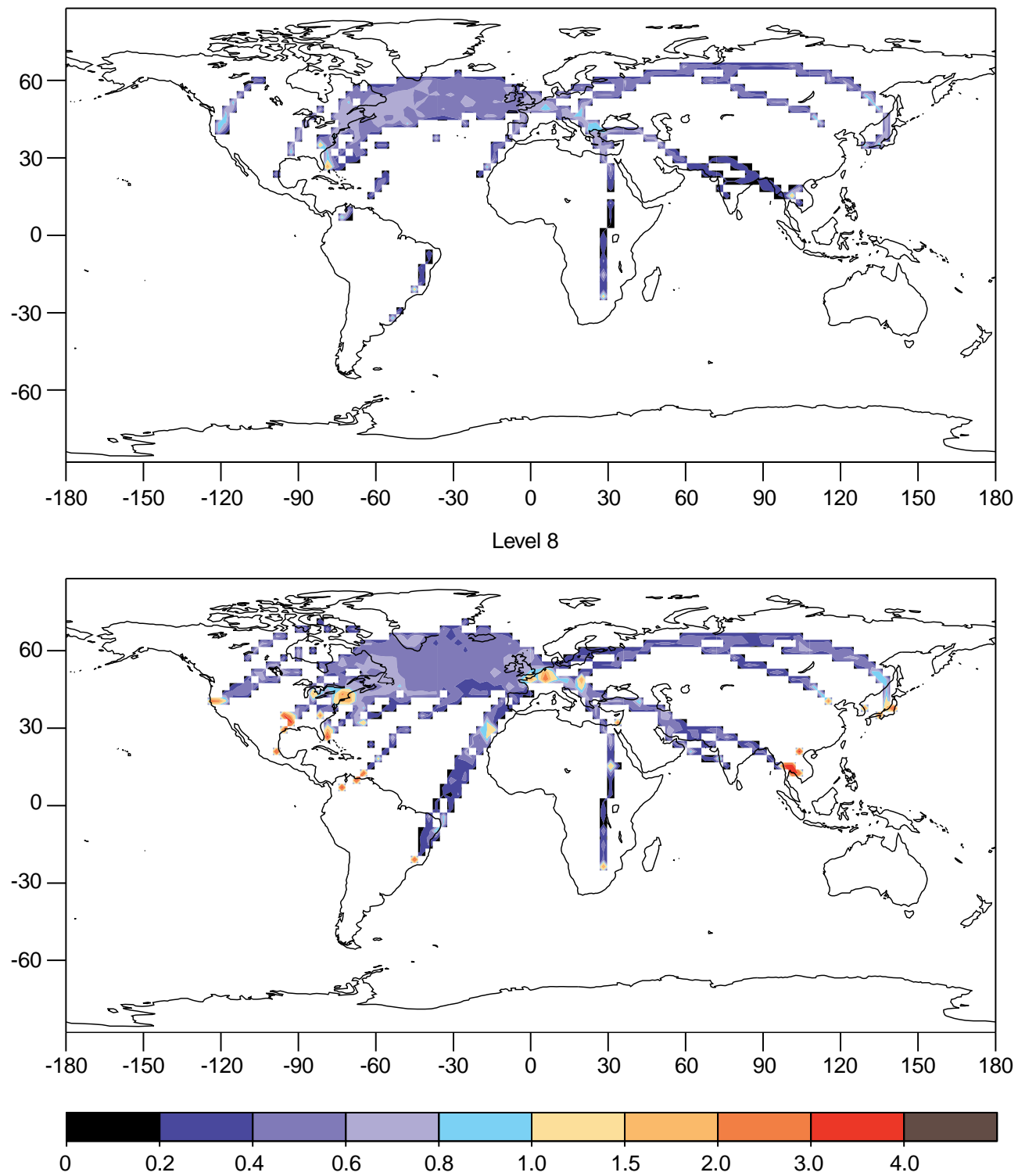

Fig. 2. Mean standard deviation of temperature in K. Mid-level pressures are $190 \mathrm{hPa}$ for level 7 and $250 \mathrm{hPa}$ for level 8 "wings" of the distribution are much broader than those of a Gaussian distribution. This finding confirms a conjecture that Sommeria and Deardorff (1977) made 20 years ago, namely that a Gaussian distribution is not appropriate to model fluctuations in a grid cell of a general circulation model. A much better fit to the fluctuation data is obtained with Lorentz curves (solid lines) $\Lambda_{\gamma}(\delta)$, i.e.

$$
\Lambda_{\gamma}(\delta)=\frac{1}{\pi} \frac{\gamma}{\delta^{2}+\gamma^{2}} .
$$

The Lorentz curve is known from spectroscopy to be the line shape of pressure broadened spectral lines. Mathematicians call it the Cauchy distribution. The parameter, $\gamma$, is the half width at half maximum (HWHM) of the distribution. By least squares fitting we found the following set of HWHMs: for level $7, \gamma_{T}=0.362 \mathrm{~K}$, $\gamma_{R H}=1.69 \%$; for level $8, \gamma_{T}=0.287 \mathrm{~K}, \gamma_{R H}=2.48 \%$. It must be admitted, that the fit using a Lorentz curve is not very good in the case of the fluctuations of relative humidities. Other distributions, such as a two-sided exponential distribution, could give better fits. However, in view of the derivation that follows, we decided to keep to the Lorentz distribution because of its highly advantageous analytical properties, which most other distributions do not share.

\section{Construction of the parametrisation}

\subsection{The joint probability for temperature and humidity fluctuations}

We derive an expression for the joint probability density (jpd), $f_{\delta T \delta R H}$, where

$$
f_{\delta T \delta R H}(\delta T, \delta R H) d \delta T d \delta R H
$$

is the probability for a simultaneous occurrence (in the same ECHAM-T42 grid cell) of a temperature and 
Level 7
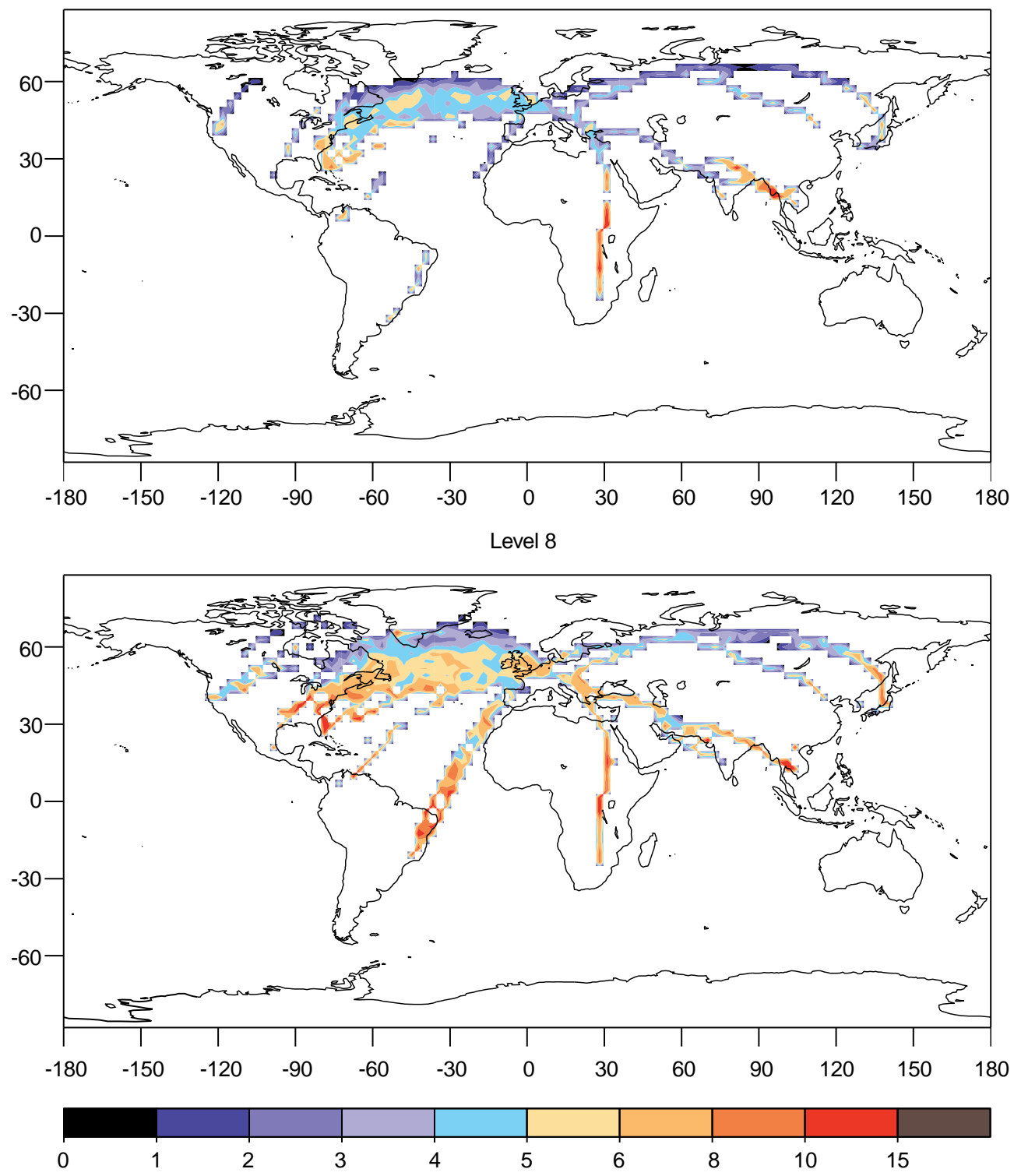

Fig. 3. Mean standard deviation of relative humidity in $\%$. Midlevel pressures are $190 \mathrm{hPa}$ for level 7 and $250 \mathrm{hPa}$ for level 8 relative humidity fluctuation in the ranges $[\delta T, \delta T+d \delta T)$ and $[\delta R H, \delta R H+d \delta R H)$, respectively. Given are the (one dimensional) probability density functions (pdf) $\varphi_{\delta T}$ and $\varphi_{\delta R H}$ which are both Lorentz distributions. If these distributions were statistically independent, we would just have to multiply them to obtain the jpd. However, $R H=e / e_{S}(T)$, so that the relative humidity fluctuation depends on the temperature fluctuation via the temperature dependence of the water vapour saturation pressure $e_{S}(T)$. For small fluctuations we may write:

$\delta R H=\frac{\partial R H}{\partial T} \delta T+\frac{\partial R H}{\partial e} \delta e=a \delta T+b \delta e$,

with

$a=-(R H \cdot L) /\left(R_{V} T^{2}\right), \quad b=1 / e_{s}(T)$.

For the derivation, the Clausius-Clapeyron equation and the ideal gas law for water vapour have been used.
From this we find that the pdf, $\varphi_{\delta R H}$, is a convolution (indicated by $\star$ ) of the following form:

$$
\begin{aligned}
\varphi_{\delta R H} & =\left[(1 / a) \varphi_{\delta T}(\delta T / a)\right] \star\left[(1 / b) \varphi_{\delta e}(\delta e / b)\right] \\
& =(1 / b) \int \varphi_{\delta T}(\delta T) \cdot \varphi_{\delta e}[(\delta R H-a \delta T) / b] d \delta T .
\end{aligned}
$$

Here we have to consider the fluctuations of the water vapour partial pressure. These have been determined in the same way as before for the fluctuations of the other variables. The distributions of $\delta e$ (dots) are displayed in Fig. 4 (lower panel) together with fitting Lorentz curves $\left(\gamma_{e}=0.068 \mathrm{~Pa}\right.$ and $0.124 \mathrm{~Pa}$ for ECHAM levels 7 and 8, respectively). Since the convolution of two Lorentzians yields another Lorentzian (this is the desired analytical property), viz.:

$\Lambda_{\gamma_{1}} \star \Lambda_{\gamma_{2}}=\Lambda_{\left(\gamma_{1}+\gamma_{2}\right)}$,

we have a consistent model insofar as we have proposed a Lorentzian distribution for the relative humidity. If we 

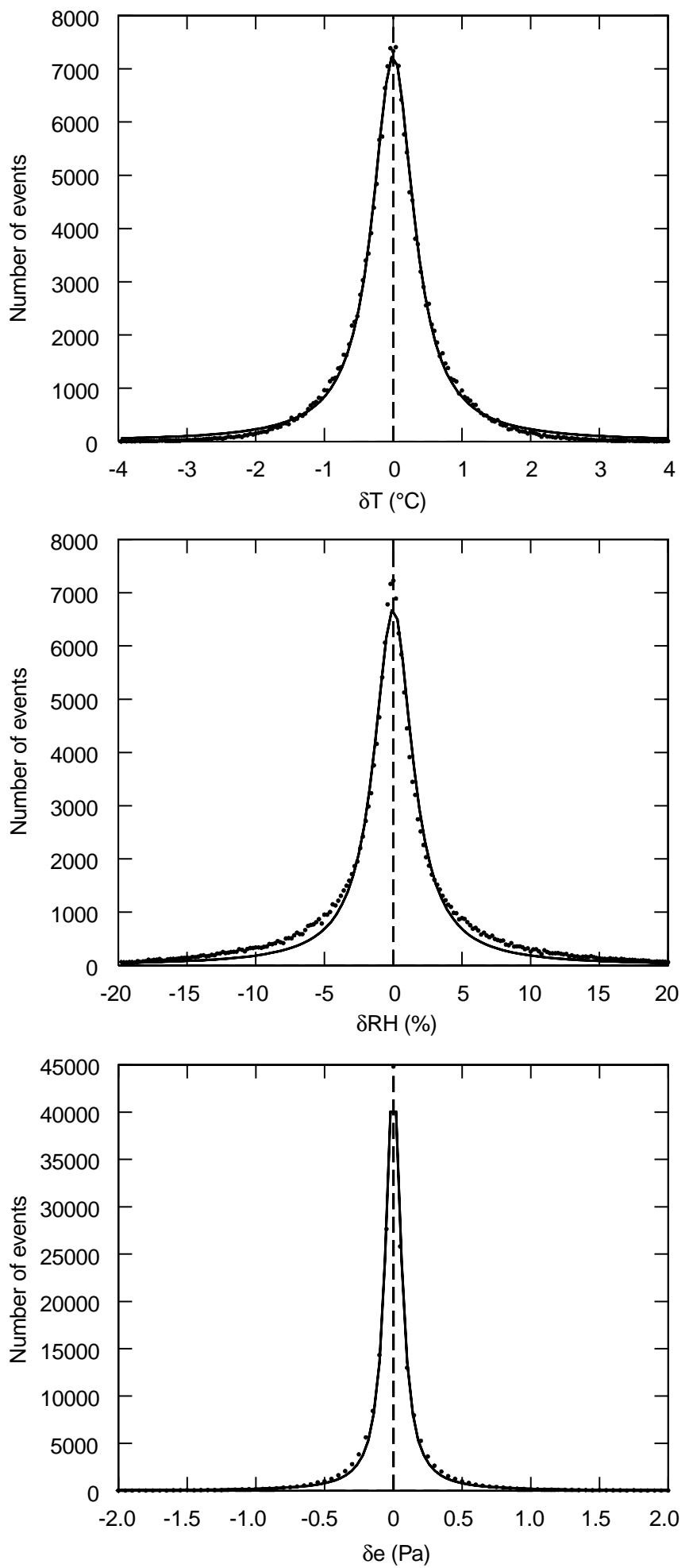

Fig. 4. Distributions (dotted) of the temperature (upper), relative humidity (middle), and water vapour partial pressure fluctuations (lower panel) for ECHAM level $7(166-222 \mathrm{hPa})$ over areas that correspond to the size of an ECHAM-T42 grid cell (i.e. about $300 \times 300 \mathrm{~km}^{2}$ ). The solid lines are Lorentz curves fitted to the distributions. The corresponding distributions for ECHAM level 8 $(222-290 \mathrm{hPa})$ are very similar had chosen another type of distribution (such as a twosided exponential), we would have to resort now to numerical methods in order to perform the convolution. For the distribution in Eq. (4) we have simply:

$(1 / a) \varphi_{\delta T}(\delta T / a)=\Lambda_{a \gamma_{T}}(\delta T)$,

$(1 / b) \varphi_{\delta e}(\delta e / b)=\Lambda_{b \gamma_{e}}(\delta e)$

The desired jpd can be written in the following form:

$f_{\delta T \delta R H}(\delta T, \delta R H) d \delta T d \delta R H=\varphi_{\delta T}(\delta T) d \delta T$.

$$
\text { - } \varphi_{\delta e}[(1 / b)(\delta R H-a \delta T)](d \delta R H / b) \text {. }
$$

Inserting the Lorentz distributions for the pdf's $\varphi$., and noting the relation $\gamma_{e}=(1 / b)\left(\gamma_{R H}-a \gamma_{T}\right)$, which follows from Eqs. (4) and (5), we find:

$$
\begin{aligned}
f_{\delta T \delta R H} & =\frac{1}{\pi^{2}} \frac{\gamma_{T}}{\delta T^{2}+\gamma_{T}^{2}} \cdot \frac{\gamma_{R H}+A \gamma_{T}}{(\delta R H+A \delta T)^{2}+\left(\gamma_{R H}+A \gamma_{T}\right)^{2}} \\
& =\Lambda_{\gamma_{T}}(\delta T) \cdot \Lambda_{\left(\gamma_{R H}+A \gamma_{T}\right)}(\delta R H+A \delta T)
\end{aligned}
$$

with $A=-a$. Please note that this function depends on the state variables $T$ and $R H$ (via $A$ ). The jpd is illustrated in Fig. 5 for two states $(T, R H)$. The shape of the jpd is discussed next.

\subsection{Formulation of the parametrisation}

Consider a certain state $\left(T_{0}, R H_{0}\right)$ in Fig. 1 to represent the actual mean values of $T$ and $R H$ in an ECHAM grid cell. The joint temperature and humidity fluctuations around the mean state $\left(T_{0}, R H_{0}\right)$ are described by the jpd, $f_{\delta T \delta R H}$, that we have derived in the previous section. A certain fluctuation $(\delta T, \delta R H)$ leads to a state in which the air can carry persistent contrails, if

$P C\left(T_{0}+\delta T, R H_{0}+\delta R H\right)=1$.

The total probability for such fluctuations is

$$
\begin{aligned}
b_{P C}\left(T_{0}, R H_{0}\right)=\iint P C & \left(T_{0}+\delta T, R H_{0}+\delta R H\right) \\
& f_{\delta T \delta R H}(\delta T, \delta R H) d \delta T d \delta R H,
\end{aligned}
$$

where the factor $A$ in the definition of $f_{\delta T \delta R H}$ has to be evaluated at $\left(T_{0}, R H_{0}\right)$. In nature, fluctuations leading to even slight water supersaturation would initiate rapid cloud formation, thereby keeping $R H$ at $100 \%$. This has been accounted for in the parametrisation by using $P C\left(T_{0}+\delta T, \widetilde{R H}\right)$ under the integral sign, with $\widetilde{R H}=$ $\min \left(R H_{0}+\delta R H, 99.9 \%\right)$. The resulting quantity, $b_{P C}$, can be interpreted to be the fraction of the cell area in which a contrail (if existent) would persist. This is the maximum possible contrail coverage. The actual contrail coverage is usually less than $b_{P C}$. For its determination measures of air-traffic density and spatial expansion of the contrails are needed. Such data cannot be obtained from the MOZAIC data bank. Hence, the task of determination of the actual contrail coverage is beyond the scope of the present study. For the calculation of $b_{P C}(T, R H)$ we have evaluated the Schmidt-Appleman criterion for pressures 190 and $250 \mathrm{hPa}$, i.e. at the mean pressures of the two considered ECHAM altitude layers. 

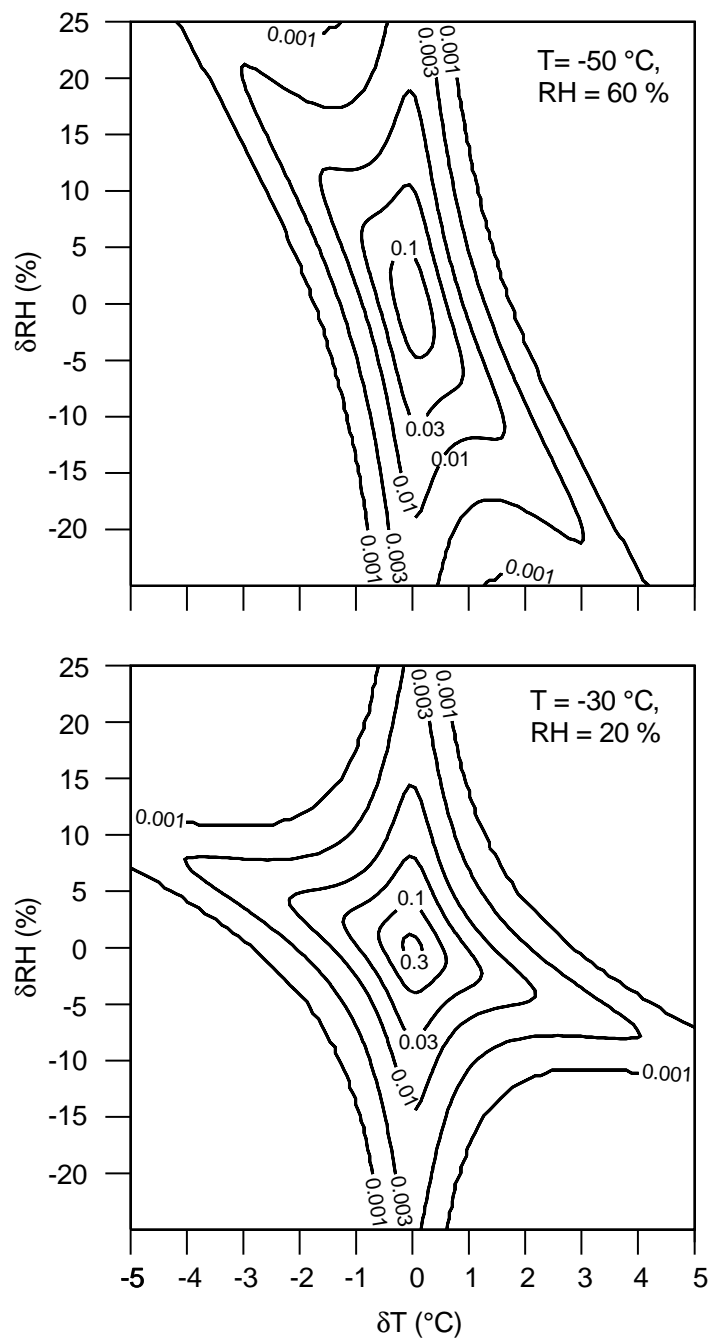

Fig. 5. Contours of the theoretical joint probability density (jpd) $f_{\delta T \delta R H}$ for common fluctuations $(\delta T, \delta R H)$ around a mean state $\left(T_{0}, R H_{0}\right)$. The jpds shown refer to $\left(-50^{\circ} \mathrm{C}, 60 \%\right)$ and $\left(-30{ }^{\circ} \mathrm{C}, 20 \%\right)$ for ECHAM level 7. The corresponding jpds for ECHAM level 8 are very similar

It has been plotted in Fig. 6. Comparison of Fig. 6 with Fig. 1 shows that $b_{P C}>0$ under conditions where the mean grid cell properties indicate no persistent contrails. It may also be noted that in the limiting cases of $P C=1$ (i.e. the lines in Fig. 1, except for the water saturation boundary) the corresponding $b_{P C}$ is 0.5 in Fig. 6 , since the fluctuations are symmetrically distributed around zero.

\section{Discussion}

\subsection{Origin of the "wings" in the fluctuation distributions}

The "wings" in the fluctuation distributions mean that large deviations from the mean values of the state variables occur with a relatively high frequency. We now discuss some possible origins of such large fluctuations. In particular, we investigate the role of (1) flight level changes, (2) tropopause penetrations, and (3) deep level convection in the tropics. Finally we show a surprising

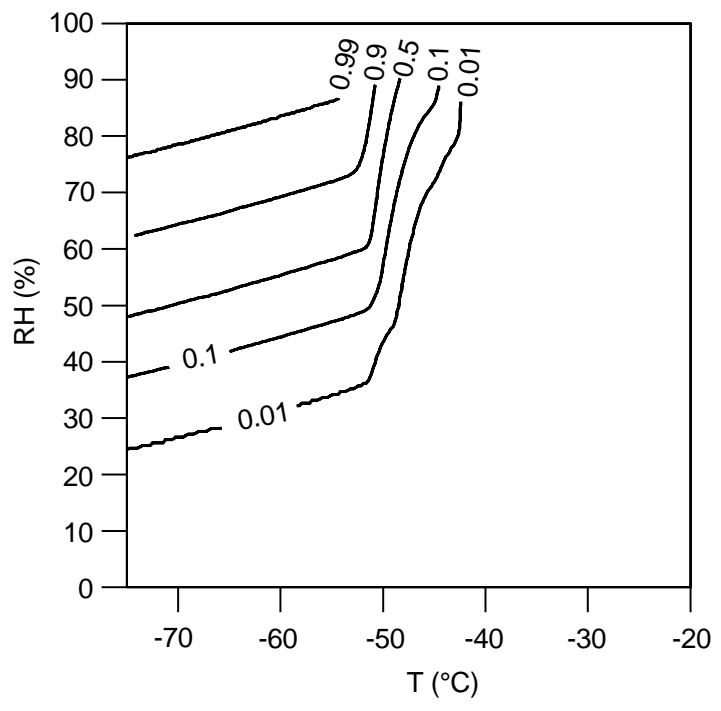

Fig. 6. Maximum possible fractional coverage of persistent contrails, $b_{P C}$, for ECHAM level 7 , as a function of the state variables temperature, $T$, and relative humidity, $R H$. The corresponding function for ECHAM level 8 is very similar

link between the large-scale meridional temperature gradient and the Lorentz distribution.

Aircraft sometimes change their flight level (i.e. altitude) while cruising. This pressure change (aircraft measure their altitude via the pressure) is usually accompanied by distinct changes in temperature and humidity. Therefore, if such a manoeuvre takes place in the middle of an ECHAM grid cell, large fluctuations should arise. Then, the determined mean values refer to some level in between the two assumed flight levels, and the actually measured values of temperature and humidity all are at a relatively large variation with these mean values. We found that flight level changes are only a minor source of large fluctuation events, both for the humidity and the temperature. Since fluctuations due to changes of the flight level are artificial and not caused by meteorological or climatic circumstances, they have been ignored in the evaluation of the MOZAIC data. The distributions presented in Sect. 2 are based on data collected at constant flight levels.

Another source of large fluctuations is penetrations of the tropopause during flight. Flight in the stratosphere is indicated in the MOZAIC data by high ozone mixing ratio and very low humidity. The temperature change during a tropopause penetration can reach $10 \mathrm{~K}$ over a distance of $200 \mathrm{~km}$. We have checked the influence of tropopause crossing on the shape of the fluctuation distributions by ignoring all data with a temperature change of more than $3 \mathrm{~K}$ within one grid cell. We found that tropopause crossings are a major cause of large humidity fluctuations $(|\delta R H| \gtrsim 5 \%)$ and a highly dominant cause of large (i.e. $|\delta T| \gtrsim 1 \mathrm{~K}$ ) temperature distributions. Fluctuations due to penetrations of the tropopause are real as they can be traced back to meteorological phenomena (e.g. upper level lows). Thus, they are contained in the distributions presented in Sect. 2. 
Restriction of the data evaluation to the tropics (i.e. south of $30^{\circ} \mathrm{N}$ ) shows that these regions contribute substantially (almost as much as tropopause crossings) to large humidity fluctuations, probably because of deep convection (see Fig. 3). On the contrary, large temperature fluctuations are caused almost exclusively outside the tropics. The reason for that is the very small meridional gradient of the zonal mean temperature over the tropics. Furthermore, the troposphere reaches very high altitudes over the tropics. Therefore tropopause penetrations rarely occur in this region.

It was shown in Sect. 2 that Lorentz distributions are well suited to fit the measured distributions of humidity and temperature fluctuations. In particular for the latter, the Lorentz formula worked very well. If data were ignored that are recorded during tropopause crossings, we still would find a Lorentz profile, though a narrower one. It is interesting to note that there is a link between the Lorentz distribution and the large-scale meridional temperature gradient. A typical meridional temperature profile of the upper troposphere of the middle latitudes and the polar front region displays a northward temperature gradient of about -0.5 to $-1 \mathrm{~K} / 100 \mathrm{~km}$ over a region of about $1000-1500 \mathrm{~km}$ width on the southern side of the jet axis. Since most of the MOZAIC flights took place over the North Atlantic a considerable number of flights were sufficiently close to the polar front jet to be inside the large-scale temperature gradient. Considering a certain ECHAM grid cell that was crossed by a large ensemble of flights during 1995, we find a corresponding number of angles, $90^{\circ}-\alpha$, between the actual flight heading and the direction of the temperature gradient. As the flights considered had different starting positions and destinations, we may consider the angle $\alpha$ a random variable. In the simplest case of a linear N-S temperature profile, the total temperature variation measured while crossing the grid cell under consideration is proportional to $\tan \alpha$. If $\alpha$ is uniformly distributed between $-90^{\circ}$ and $+90^{\circ}$, then the temperature variation will be distributed like $\tan \alpha$, and this is a Lorentz (or Cauchy) distribution (see, e.g. Goodman, 1988, p. 62). In spite of the somewhat idealistic assumptions in these arguments we think that the large-scale meridional temperature gradient at least establishes a strong tendency towards a Lorentz distribution.

\subsection{Check of the joint probability density}

In Sect. 2c we derived a theoretical expression for the joint probability density (jpd) of common temperature and humidity fluctuations. This function depends on the mean state, say $\left(T_{0}, R H_{0}\right)$, via the factor $A$ which depends on the state variables. Next we need to check whether the theoretical jpd is a good model for the fluctuations $(\delta T, \delta R H)$ that can be obtained from the data bank. To this end, we determine the frequencies of fluctuations in finite ranges $\Delta T \times \Delta R H$, with bin widths $\Delta T=0.1 \mathrm{~K}$ and $\Delta R H=0.5 \%$ and plot the result in Fig. 7 .
Comparison between Figs. 5 (theoretical jpds) and 7 (empirical jpd) shows that the empirical jpd is broader and rounder than the theoretical jpds. Whereas in the theoretical jpds the outer contours are strongly curved, this is not so in the empirical jpd. In both jpds we observe two "main axes", which correspond to $\delta T \approx 0$ (vertical axis) and $\delta e \approx 0$ (the oblique axis, i.e. fluctuation of $R H$ induced by a pure temperature fluctuation, without a fluctuation of the absolute humidity). The angle between the main axes depends on the mean state $\left(T_{0}, R H_{0}\right)$. The first impression is that the angle between the main axes is rather large in the empirical jpd, which resembles rather the shape of the theoretical jpd for $\left(-30^{\circ} \mathrm{C}, 20 \%\right)$ than that for $\left(-50{ }^{\circ} \mathrm{C}, 60 \%\right)$ where the oblique axis is much steeper. The first of these states would, however, imply a much lower mean contrail frequency than is actually observed. Do these differences mean that the theoretical jpd is a poor model of the actual fluctuations? We don't think so. We rather think that the empirical jpd is a weak representation of the
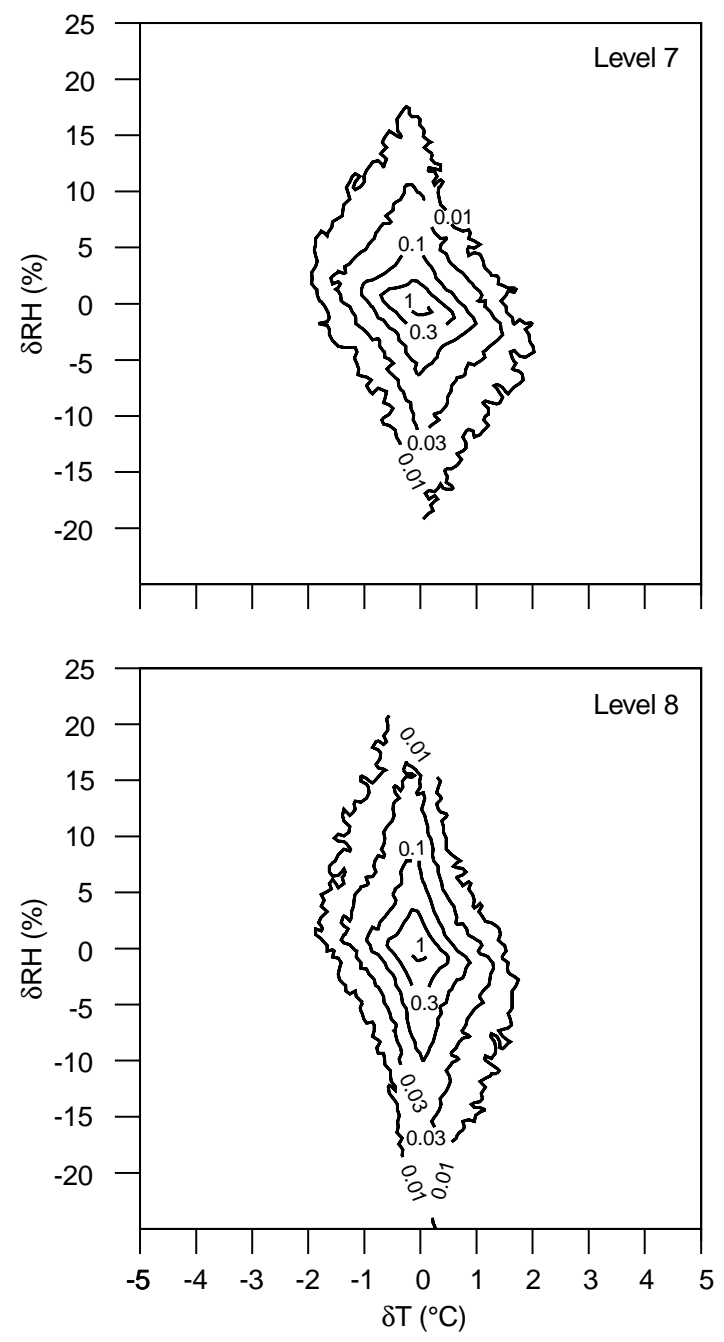

Fig. 7. Relative frequency of common fluctuations of relative humidity, $\delta R H$, and temperature, $\delta T$. This frequency distribution may be conceived an empirical jpd of common temperature and humidity fluctuations as obtained from the data from the MOZAIC data bank. The normalisation is as in Fig. 5 . 
truth, because for its determination we had to pile up all data, disregarding the different mean state variables. Thus, the empirical jpd of Fig.7 is a mixture of jpds for a large number of mean states. The mixing obviously leads to rounder contours. Remember the central limit theorem: in the limit of mixing more and more distributions of the same type one ends up with a Gaussian distribution. The product of two Gaussian distributions possesses elliptic contours. The angle between the two main axes of the jpds is a function of $A$ which, in turn, depends on the mean state $\left(T_{0}, R H_{0}\right)$. Mixing of the states thus yields a mixture of $A$-values, which makes the angle between the two axes unclear (i.e. the oblique axis is not well defined, which also corresponds to the rounding of the contours). As a further test we picked out all fluctuation data that belong to mean values in a small interval $\left(T_{0} \pm 0.5 \mathrm{~K}, R H_{0} \pm 2.5 \%\right)$, where $T_{0}$ and $R H_{0}$ were chosen such that as large as possible number of events could be obtained. Although the resulting empirical jpd is rather noisy in this constrained ensemble, it agrees reasonably well with the corresponding theoretical jpd, or at least no striking differences are visible (Fig. 8).

Furthermore, there is a weak positive correlation between fluctuations of water vapour partial pressure, $\delta e$, and temperature, $\delta T$ (Fig. 9). This has not been taken into account in the derivation of the theoretical jpd. Such a correlation can turn the $\delta e \approx 0$ axis towards the horizontal direction. (A positive correlation between $\delta e$ and $\delta T$ would lead to a reduction of the factor $A$ in the theoretical derivation.) The correlation between $\delta e$ and $\delta T$ can probably be explained using the following

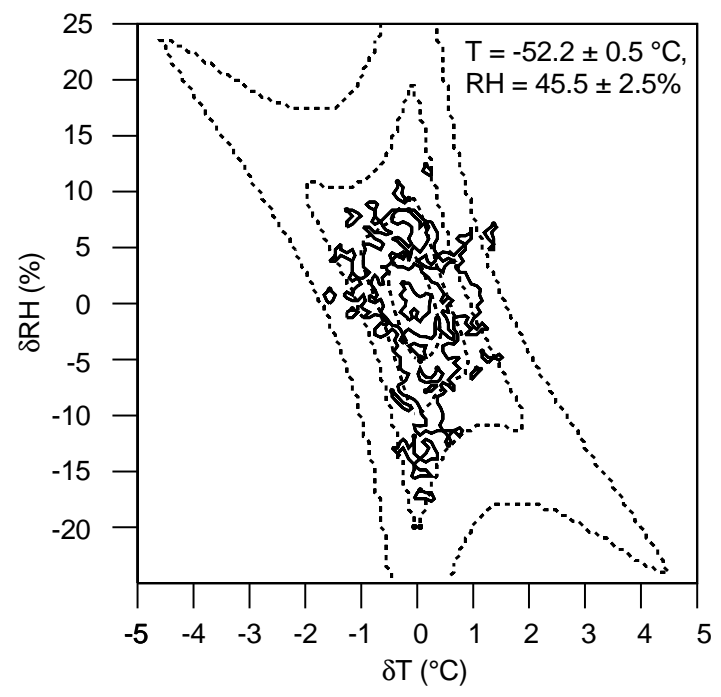

Fig. 8. Direct comparison of a (non-normalized) empirical jpd (solid) with a corresponding theoretical jpd (dashed) for a constrained data base. The fluctuation data in the empirical jpd refer to mean values in the small intervals indicated along the top border of the Fig., the theoretical jpd is drawn for the central state in this interval. The empirical jpd is rather noisy, because the data base is small. Contour values are 1, 3 and 10 events for the empirical jpd, and 0.001, 0.003, 0.01 , and 0.03 for the theoretical jpd. The empirical jpd has not been normalised because the statistical errors are large and a normalisation would pretend only small statistical errors
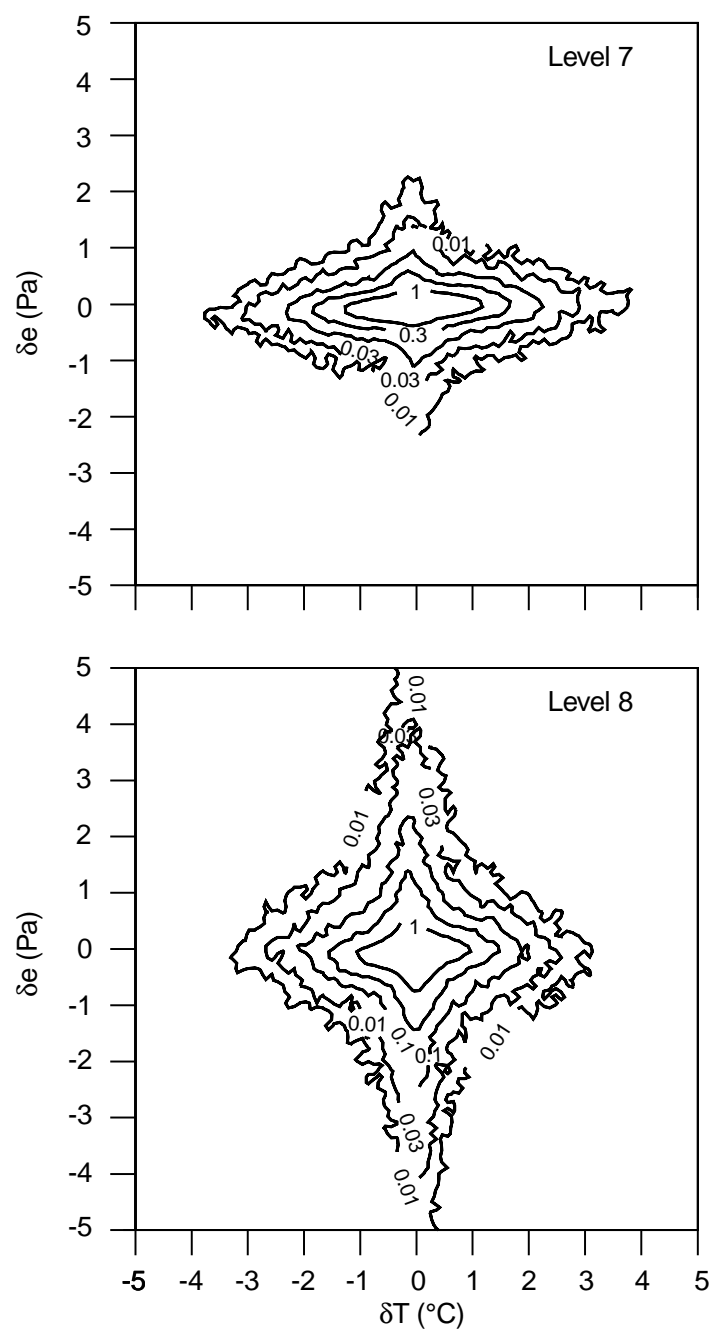

Fig. 9. Relative frequency of common fluctuations of water vapour partial pressure, $\delta e$, and temperature, $\delta T$ based on the MOZAIC data bank. The horizontal "axis" of the frequency distribution is slightly tilted which means a weak positive correlation between $\delta e$ and $\delta T$

meteorological argument: often it gets warmer when air is advected from the south. Air from southern regions is usually moister than air from other directions. Hence it usually becomes moister when it gets warmer (at least on a synoptic scale). We have neglected this weak correlation in the derivation of the jpd because there is no physical relation between $\delta e$ and $\delta T$ and we did not want to introduce empirical relations. Anyway, the possible error that the neglect of this correlation causes in the final result, viz. the maximum contrail coverage $b_{P C}$, is probably small when compared to the errors that arise in the determination of the actual contrail coverage from uncertainties in air traffic densities and contrail spreading rates.

\subsection{Influence of data quality on the parametrisation}

Finally, let us discuss the influence of possible systematic errors in the humidity data on the derived parametrisation. If such a bias were constant on time scales longer than $30 \mathrm{~min}$, it would not influence the parametrisation 
because it would then have no effect on the fluctuation data. Such a bias would only yield incorrect mean values, yet these are not used for the parametrisation. If, on the other hand, such an error possessed a more random nature, i.e. if its time scale were less than $30 \mathrm{~min}$, it would corrupt the fluctuation data and produce broader distributions.

However, it turned out that the widths of the distributions only weakly affect the resulting parametrisation, viz. the function $b_{P C}$. This has been checked as follows: instead of fitting the fluctuation data with a simple least squares fit, we fitted them also using a weighted least squares criterion, with weights $1 / n_{i}$ where $n_{i}$ is the number of events. This fitting yielded Lorentzians with a different set of half widths: for level $7, \gamma_{T}=0.292 \mathrm{~K}, \gamma_{R H}=2.07 \%$; for level $8, \gamma_{T}=0.216 \mathrm{~K}$, $\gamma_{R H}=3.18 \%$. The resulting function $b_{P C}$ (not shown) for this set of HWHMs is little different from the one shown in Fig. 6. Thus, we may conclude that our parametrisation is rather stable against small errors in the determination of the fluctuation distributions.

\section{Summary}

We have evaluated humidity and temperature data from MOZAIC for the aim of a parametrisation of persistent contrail coverage for global circulation models like ECHAM. The derived parametrisation is the function $b_{P C}(T, R H)$ (Sect. 3) as plotted in Fig. 6, which depends on the prognostic state variables temperature and relative humidity and which can be implemented into GCMs, e.g. as a table or an approximative function. $b_{P C}$ is the fraction of the volume of a grid cell in which contrails, if present, would persist. How much of this fraction actually contains contrails depends on the air traffic density and on the spatial expansion and duration of the contrails. Generally, the actual contrail coverage will be less than $b_{P C}$. Thus, $b_{P C}$ primarily characterises the existence and expansion of the "moist lenses" in which contrails occur in groups (Bakan et al., 1994).

As a further result of the MOZAIC data evaluation we could determine quantitatively temperature and humidity fluctuations on a $300 \times 300 \mathrm{~km}^{2}$ scale. As far as we are aware, this has been done here for the first time, since data sets with such an extensive spatial and temporal coverage like MOZAIC were not available before. We showed that fluctuations on a large scale cannot be modelled by Gaussian distributions. Instead they must be modelled using distributions with extended wings, because large deviations of a quantity from its large-scale average have a relatively high probability. So far, cloudiness in general circulation models has been modelled using ad hoc assumptions about the distribution of temperature and humidity fluctuations (e.g. Smith 1990; Ricard and Royer 1993). Here we offer the possibility to set up such a parametrisation on an observationally justified basis.

Acknowledgement. The MOZAIC project is funded by the Commission of the European Union (contract AERO-CT920052) and coordinated by A. Marenco of CNRS in Toulouse. Part of the work has been supported within the BMBF-project "Schadstoffe in der Luftfahrt" (contract 01LL9602-9). We thank two anonymous reviewers for their useful criticism.

Topical Editor L. Eymard thanks S. Bakan and another referee for their help in evaluating this paper.

\section{References}

Appleman, H., The formation of exhaust condensation trails by jet aircraft, Bull. Am. Meteorol. Soc., 34, 14-20, 1953.

Bakan, S., M. Betancor, V. Gayler, and H. Graßl, Contrail frequency over Europe from NOAA-satellite images, Ann. Geophysicae, 12, 962-968, 1994.

Busen, R., and U. Schumann, Visible contrail formation from fuels with different sulfur contents, Geophys. Res. Lett., 22, 13571360, 1995.

Fortuin, J. F. P., R. van Dorland, W. M. F. Wauben, and H. Kelder, Greenhouse effects of aircraft emissions as calculated by a radiative transfer model, Ann. Geophysicae, 13, 413-418, 1995.

Goodman, R., Introduction to Stochastic Models, Benjamin/Cummings, Menlo Park, California, 1988

Graßl, H., Possible climate effects of contrails and additional water vapor, in Air-Traffic and the Environment, Ed. U. Schumann, Lecture Notes in Engineering, 60, Springer, Berlin Heidelberg New York, 124-137, 1990.

Holton, J. R., An Introduction to Dynamic Meteorology, 2nd edn, Academic Press, New York, 1979.

Marenco, A., P. Nedelec, V. Thouret, and C. Grouhel, Measurement of ozone and water vapour on airbus in-service aircraft: the Mozaic programme, in Impact of Emissions from Aircraft and Spacecraft Upon the Atmosphere, Eds. U. Schumann and D. Wurzel, DLR-Mitteilung 94-06, 26-31, 1994.

Ponater, M., S. Brinkop, R. Sausen, and U. Schumann, Simulating the global atmospheric response to aircraft water vapour emissions and contrails - a first approach using a GCM, Ann. Geophysicae, 14, 941-960, 1996.

Ricard, J. L., and J. F. Royer, A statistical cloud scheme for use in an AGCM, Ann. Geophysicae, 11, 1095-1115, 1993.

Roeckner, E., K. Arpe, L. Bengtsson, S. Brinkop, L. Dümenil, M. Esch, E. Kirk, F. Lunkeit, M. Ponater, B. Rockel, R. Sausen, U. Schlese, S. Schubert, M. Windelband, Simulation of the present-day climate with the ECHAM model: impact of model physics and resolution, Max-Planck-Institut für Meteorologie, Report 93, 171 pp., Hamburg, 1992.

Schmidt, E., Die Entstehung von Eisnebel aus den Auspuffgasen von Flugmotoren, Schriften der Deutschen Akademie der Luftfahrtforschung, Verlag R. Oldenbourg, München und Berlin, H. 44, $1-15,1941$.

Schumann, U., On conditions for contrail formation from aircraft exhausts, Z. Meteorol., N. F., 5, 4-23, 1996.

Smith, R. N. B., A scheme for predicting layer clouds and their water content in a general circulation model, Q.J. R. Meteorol. Soc., 116, 435-460, 1990.

Sommeria, G., and J. W. Deardorff, Subgrid-scale condensation in models of non-precipitating clouds, J. Atmos. Sci., 34, 344-355, 1977. 